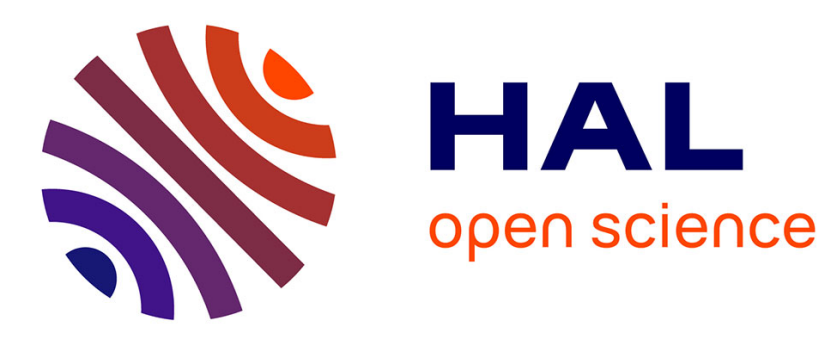

\title{
Radiative Thermal Memristor
}

Jose Ordonez-Miranda, Younes Ezzahri, Jose Tiburcio-Moreno, Karl Joulain, Jérémie Drevillon

\section{To cite this version:}

Jose Ordonez-Miranda, Younes Ezzahri, Jose Tiburcio-Moreno, Karl Joulain, Jérémie Drevillon. Radiative Thermal Memristor. Physical Review Letters, 2019, 123 (2), 10.1103/PhysRevLett.123.025901 . hal-02332163

\section{HAL Id: hal-02332163 https://hal.science/hal-02332163}

Submitted on 21 Nov 2020

HAL is a multi-disciplinary open access archive for the deposit and dissemination of scientific research documents, whether they are published or not. The documents may come from teaching and research institutions in France or abroad, or from public or private research centers.
L'archive ouverte pluridisciplinaire HAL, est destinée au dépôt et à la diffusion de documents scientifiques de niveau recherche, publiés ou non, émanant des établissements d'enseignement et de recherche français ou étrangers, des laboratoires publics ou privés. 


\title{
Radiative Thermal Memristor
}

\author{
Jose Ordonez-Miranda, ${ }^{1, *}$ Younès Ezzahri, ${ }^{1}{ }^{\dagger}{ }^{\dagger}$ Jose A. Tiburcio-Moreno, ${ }^{2}$ Karl Joulain, ${ }^{1}$ and Jérémie Drevillon ${ }^{1}$ \\ ${ }^{1}$ Institut Pprime, CNRS, Université de Poitiers, ISAE-ENSMA, F-86962 Futuroscope Chasseneuil, France \\ ${ }^{2}$ Universidad Nacional Jorge Basadre Grohmann, Facultad de Ciencias, \\ Av. Miraflores s/n, Ciudad Universitaria, Tacna, Perú
}

(Dated: October 24, 2019)

\begin{abstract}
Based on the thermal hysteresis of a phase change material exchanging radiative heat with a phase invariable one, we propose a radiative thermal memristor characterized by a Lissajous curve between their exchanged heat flux and temperature difference periodically modulated in time. For a memristor with terminals of $\mathrm{VO}_{2}$ and a blackbody, it is shown that: (i) the temperature variations of its memristance follow a closed loop determined by the thermal hysteresis width of $\mathrm{VO}_{2}$. (ii) The thermal memristance on/off ratio is determined by the contrast of $\mathrm{VO}_{2}$ emissivities for its insulating and metallic phases and is equal to 3.59. The analogy of the proposed memristor to its electrical counterpart makes it promising to lay the foundations of the thermal computing with photons.
\end{abstract}

PACS numbers: 65.60.+a; 65.80.-g; 65.90.+i

The last fifteen years have witnessed the emergence of the new research area thermotronics devoted to the development of thermal analogues of the fundamental electronic components, by means of the heat transport via phonons, electrons, and photons [1-12]. Several conceptual devices, such as thermal rectifiers/diodes [13-19], thermal transistors [20-26], thermal logical gates [27] and thermal memories [28] have been proposed and the working principles of some of them have experimentally been proven[29-31], which makes it possible to process information through heat currents.

The electrical memristor, a two-terminal device relating electrical charge with magnetic flux in electronics circuits, has recently attracted an enormous interest, due to its tremendous and revolutionary potential in microelectronics, informatics, spintronics, and neuromorphic networks [9, 32]. Based on symmetry arguments, Chua [33] first conceived this device in 1971 as the fourth fundamental electronic component besides the resistor, capacitor and inductor. Almost forty years later, Strukov et al. [34] developed the physical model for describing the hysteretic current-voltage behavior that characterizes an electrical memristor and showed that its realization can be achieved with doped metal-oxide semiconductors.

Despite of the vast research on and development of the electrical memristor [32], its thermal counterpart has received little attention. Based on the asymmetric temperature response of the thermal conductivity of $\mathrm{VO}_{2}$ during its heating and cooling, Ben-Abdallah [35] proposed a conductive thermal memristor, whose Lissajous curve between the heat flux and temperature difference of its terminals was recently reported by Yang et al. [36]. The operation of this promising memristor is driven by the hysteretic metal-insulator transition of $\mathrm{VO}_{2}$, which is one of the most investigated Phase Change Materials (PCM), due to its ability to change its thermal, optical, and electrical properties in a narrow interval of temperatures, near room temperature [37-41]. The performance of the conductive memristor is, however, limited by the inevitable presence of interface thermal resistances and the speeds of the involved phonons and electrons, which are typically 3 orders of magnitude smaller than that of photons. These limitations could thus be overcome by a radiative memristor with contactless terminals.

The purpose of this letter is to demonstrate the proof of principle of a radiative thermal memristor, an anologue of the electrical memristor defined by the functional relation $T=M(x, q) q$ between the temperature difference $(T)$ and heat flux $q$ of a system with a generalized thermal resistance $M(x, q)$ determined by $q$ and a state variable $x$ driving the internal state of the system [33, 34]. The $q-T$ characteristic curve of this memristive system is generally a Lissajous figure crossing the origin $(q=0$ for $T=$ $0)$, which distinguishes it from an arbitrary dynamical system, as is the case of an electrical memristor [34].

Let us consider a PCM exchanging heat by far-field thermal radiation with a non-PCM, due to their temperature difference $\Delta T(t)$ periodically modulated in time $t$, as shown in Fig. 1. The emissivity $\varepsilon_{1}$ of the PCM depends on its temperature $T_{0}+\Delta T(t)$, while the one $\varepsilon_{2}$ of the non-PCM is considered to be independent of its corresponding steady-state temperature $T_{0}$, as is the case of $\mathrm{SiO}_{2}$ and $\mathrm{SiC}$, for $T_{0}<600 \mathrm{~K}$ [42]. In order to ensure the thermalization of the PCM, we consider that the modulation period $\tau$ of $\Delta T(t)$ is much longer than its thermalization time (time required to reach the thermal equilibrium between its electrons and phonons), which tipically ranges from a few to a hundred microseconds, for the case of $\mathrm{VO}_{2}[37,43]$ used in this work. This condition is good enough to guarantee that the heating frequency $\left(\tau^{-1} \lesssim 1 \mathrm{MHz}\right)$ is much smaller than the spectral frequencies of the photons emitted by the two materials, which are in the order of $\mathrm{GHz}$, for temperatures near room temperature [44]. Under this quasi-steady state, the far-field heat flux $q$ exchanged by the two plates is 


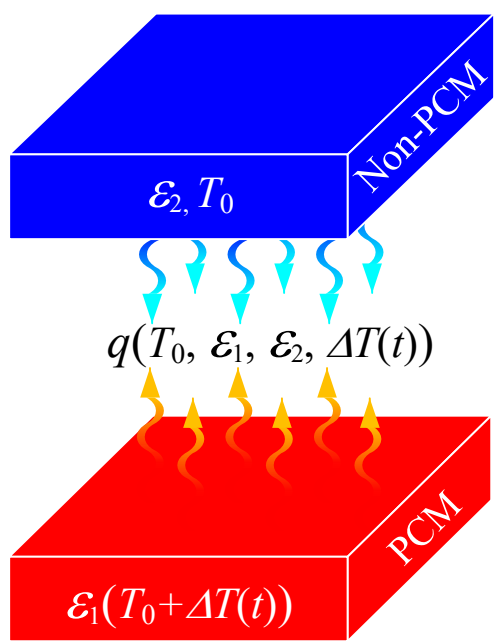

FIG. 1. Scheme of a radiative thermal memristor: a PCM exchanging radiative heat with a non-PCM through a vacuum gap, due to their temperature difference $\Delta T(t)$ periodically modulated in time $t$.

given by the Stefan-Boltzmann law, as follows [44]

$$
q=\sigma \varepsilon\left(T_{0}+\Delta T(t)\right)\left[\left(T_{0}+\Delta T(t)\right)^{4}-T_{0}^{4}\right],
$$

where $\sigma$ is the Stefan-Boltzmann constant and the effective emissivity $\varepsilon=\left(\varepsilon_{1}^{-1}+\varepsilon_{2}^{-1}-1\right)^{-1}$ is determined by the emissivities of the PCM and non-PCM [44]. For the sake of simplicity, we will consider that this non-PCM is a blackbody $\left(\varepsilon_{2}=1\right)$, which reduces the effective emissivity to that of the $\operatorname{PCM}\left(\varepsilon=\varepsilon_{1}\right)$.

We consider that the emissivity $\varepsilon$ of the PCM changes for temperatures within its phase transition, such that its value $\left(\varepsilon_{h}\right)$ during the heating process is different than that $\left(\varepsilon_{c}\right)$ for the cooling one, at a given temperature $T$, as is the case of $\mathrm{VO}_{2}[37,45-47]$. This thermal hysteresis of the PCM emissivity is driven by correlated atomic interactions and establishes that $\varepsilon$ is given by

$$
\varepsilon(T)=\varepsilon_{h}(T) \delta(T \uparrow)+\varepsilon_{c}(T) \delta(T \downarrow),
$$

where $\delta(T \uparrow)$ and $\delta(T \downarrow)$ take the value of unity as the PCM temperature raises and lowers, respectively, otherwise they reduce to zero. For $\mathrm{VO}_{2}$, the emissivitities $\varepsilon_{h}$ and $\varepsilon_{c}$ can be well described by the following model [48]

$$
\varepsilon_{n}(T)=\varepsilon_{i}+\frac{\varepsilon_{m}-\varepsilon_{i}}{1+e^{-\beta\left(T-T_{0 n}\right)}},
$$

where $\varepsilon_{i}=0.79$ and $\varepsilon_{m}=0.22$ are the emissitivities of $\mathrm{VO}_{2}$ in its insulating and metallic phases, respectively, $T_{0 h}=342.5 \mathrm{~K}$ and $T_{0 c}=334.5 \mathrm{~K}$ are its respective transition temperatures for the heating $(n=h)$ and cooling $(n=c)$ processes, and $\beta=1.57 \mathrm{~K}^{-1}$. Equations (2) and (3) thus take into account the $\mathrm{VO}_{2}$ thermal hysteresis of $T_{0 h}-T_{0 c}=8 \mathrm{~K}$ experimentally observed by Qazilbash et al.[40] and they indicate that the $\mathrm{VO}_{2}$ emissivity is bounded between its values for the metallic and insulating phases $\left(\varepsilon_{m} \leq \varepsilon_{1} \leq \varepsilon_{i}\right)$, as shown in Fig. 2. Note that at low temperature $(T<332 \mathrm{~K}), \mathrm{VO}_{2}$ behaves as a insulator with high emissivity, while for high temperatures $(T>345 \mathrm{~K})$, it becomes a metal with low emissivity, such that its emissivity changes a factor of $\varepsilon_{i} / \varepsilon_{m}=3.59$ across its phase transition. This significant emissivity contrast in a relatively small temperature interval, allows to enhance (reduce) the radiative heat flux with $\mathrm{VO}_{2}$ in its insulating (metallic) phase, as established by Eq. (1).

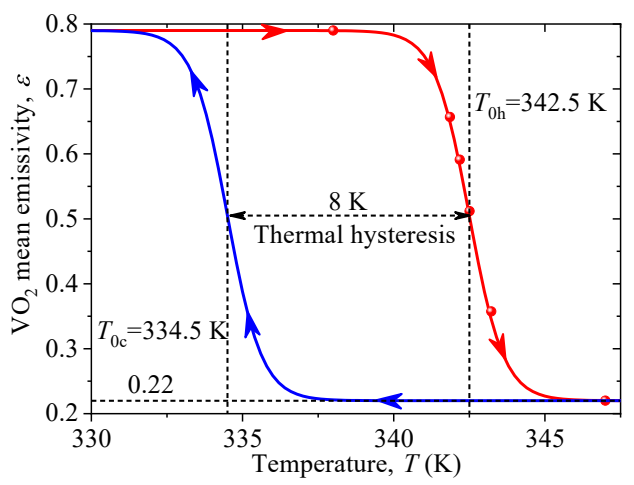

FIG. 2. Mean emissivity of $\mathrm{VO}_{2}$ for its heating and cooling processes. The dots stand for experimental data [40, 41, 48], while the lines represent the predictions of Eq. (3).

According to Eq. (1), the emissitivy variations of $\mathrm{VO}_{2}$ between its insulating and metallic phases along with its thermal hysteresis generate a closed loop for the evolution of heat flux $q$, as the temperature difference $\Delta T$ periodically changes through time. This loop unfolds in two coupled loops (Lissajous curve) by properly modulating $\Delta T$ through positive and negative values, as shown below. The effective thermal resistance $\Delta T / q$ of the system (Fig. 1) will thus depends on $\Delta T$ and its sign (heating or cooling), which represents the history of the input temperature difference. This thermal memory effect is analogous to that of an electrical memristor characterized by a Lissajous $I-V$ curve that induces the dependence of its electrical resistance on the input voltage difference and its polarity [34]. The radiative system shown in Fig. 1 can thus play the role of a thermal memory resistor (thermal memristor) with a memory resistance (memristance) $M=\Delta T / q$ given by Eq. (1), as follows

$$
M^{-1}=\sigma \varepsilon\left(T_{0}+\Delta T\right)\left(2 T_{0}+\Delta T\right)\left[\left(T_{0}+\Delta T\right)^{2}+T_{0}^{2}\right] .
$$

Taking into account that the PCM temperature $T_{0}+\Delta T$ determining the values of $M$ also drives the appearance of metallic (insulating) domains in an insulating (a metallic) matrix during the heating (cooling) processes [37], the memristance can also be described in terms of the volume fraction of these microscopic domains $[40,41]$. For instance, the volume fraction $f_{h}$ of the metallic domains appearing during the heating 
of $\mathrm{VO}_{2}$ at temperature $T$ is given by [37] $f_{h}(T)=$ $(-T / U) W_{0}\left\{-(U / 2 T) \exp (-U / T) \operatorname{ercf}\left[2 \beta\left(T_{0 h}-T\right) / 5\right]\right\}$, where $W_{0}($.$) is the main branch of the Lambert \mathrm{W}$ function, erfc(.) is the complementary error function and $U$ is the activation energy (in units of Kelvin) of the metallic domains. Taking into account that $U \ll T$ [37], for all temperatures within the $\mathrm{VO}_{2}$ phase transition, and that $W_{0}(x \ll 1) \approx x$, this later relation reduces to $f_{h}(T) \approx$ $0.5 \operatorname{ercf}\left[2 \beta\left(T_{0 h}-T\right) / 5\right] \approx\left[1+\exp \left(-\beta\left(T-T_{0 h}\right)\right)\right]^{-1}$, which in combination with Eq. (3), yields

$$
f_{h}(T)=\frac{\varepsilon_{i}-\varepsilon_{h}(T)}{\varepsilon_{i}-\varepsilon_{m}} .
$$

Equation (5) thus relates the microstructural parameter $f_{h}$ of $\mathrm{VO}_{2}$ with its emissivity $\varepsilon_{h}$ for the heating process. For the cooling process, on the other hand, one can show that the known volume fraction $f_{c}$ [37] of the metallic matrix of $\mathrm{VO}_{2}$ containing its insulating domains, also reduces to Eq. (3), provided that $U \ll T$ and the subindex $h$ is replaced by $c$. The common dependence of $f_{n}, \varepsilon_{n}$ and $M$ on the PCM temperature $T_{0}+\Delta T$ allows therefore to consider the ensemble of Eqs. (2)-(5) as a system of parametric equations for the evolution of the thermal memristance $M(f)$ with the volume fraction $f=f_{h} \delta(T \uparrow)+f_{c} \delta(T \downarrow)$ describing the degree of metallization of $\mathrm{VO}_{2}$, across its phase transition. This control of the thermal memristance by the state variable $f$ reinforces its analogy with the electrical memristance, whose values are driven by the electrical charge[34], which is a state variable in electricity.

The heat flux and memristance are now numerically analyzed for a thermal memristor made up of a $\mathrm{VO}_{2}$ plate exchanging radiative heat with a blackbody. To capitalize on the thermal hysteresis of the $\mathrm{VO}_{2}$ emissivity, the reference temperature $T_{0}=\left(T_{0 h}+T_{0 c}\right) / 2=338.5 \mathrm{~K}$ is set as the average of the insulating-to-metal and metalto-insulating transition temperatures. Furthermore, we consider a temperature difference $\Delta T(t)=\theta \sin (2 \pi t / \tau)$ varying sinusoidally with time, in a similar way to the electrical memristor, which is usually excited with an alternating voltage difference $[33,34]$. The amplitude $\theta$ is chosen large enough $\left(\theta \geq T_{0 h}-T_{0 c}=8 \mathrm{~K}\right)$ to ensure that $\mathrm{VO}_{2}$ completes its hysteresis loop after one period $\tau$. In practice, this periodic oscillation of the PCM temperature can be set with a Peltier cell [49], while the non-PCM is kept at constant temperature with a second Peltier cell. These cells typically operate with a resolution better than $0.1 \mathrm{~K}$ [50] and modulation frequencies from $0.1 \mathrm{~Hz}$ up to $100 \mathrm{~Hz}$, which are much smaller than the infrared ones driving the thermal radiation at temperatures around $T_{0}$. This is the reason why the oscilating temperature of the PCM is not expected to significantly alter the equilibrium state of the emitted photons in the radiative thermal memristor shown in Fig.1.

Figure 3 shows the heat flux $q$, temperature difference $\Delta T$ and $\mathrm{VO}_{2}$ emissivity $\varepsilon$ driving the thermal radiation in

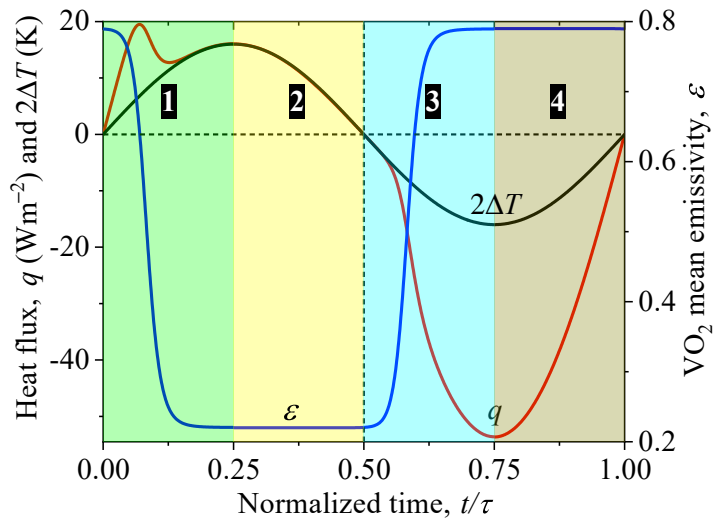

FIG. 3. Time evolution of the heat flux, temperature difference and $\mathrm{VO}_{2}$ emissivity driving the thermal radiation in the memristor shown in Fig. 1. The colored zones 1 and 3 stand for phase transitions, while the zones 2 and 4 represent no phase changes. Calculations were done for $\theta=8 \mathrm{~K}$.

the thermal memristor, as functions of time. Note that $\varepsilon$ exhibits four different behaviors, within one period $\tau$. In the initial case $1(0 \leq t / \tau<0.19), \varepsilon$ drastically reduces due to the insulating-to-metal transition of $\mathrm{VO}_{2}$, as $\Delta T$ increases. An additional increase and then decrease of $\Delta T$ keeps the $\mathrm{VO}_{2}$ in its metallic phase with constant and low emissivity, as revealed by case $2(0.19 \leq t / \tau<0.50)$. In case $3(0.50 \leq t / \tau<0.69), \Delta T$ continues reducing and triggers the metal-to-insulating transition of $\mathrm{VO}_{2}$ characterized by the significant increases of $\varepsilon$, as shown in Fig. 2. The subsequent variations of $\Delta T$ maintain the $\mathrm{VO}_{2}$ in its insulating phase with constant and high emissivity, as displayed by case $4(0.69 \leq t / \tau<1)$. The combined time variations of $\Delta T$ and $\varepsilon$ for cases 1 and 3 , induce a heat flux with a strong nonlinear dependence on the temperature difference, despite of the fact that $\Delta T \ll T_{0}=338.5$ $\mathrm{K}$. For cases 2 and 4 , on the other hand, $\varepsilon$ takes constant values and $q \propto \Delta T$, as shown in Fig. 3 by the occurrence of the maximum and minimun values $q$ and $\Delta T$ at the same times, and is established by Eq. (1), for $\Delta T \ll T_{0}$. It is thus clear that the sharp variations of the heat flux with respect to those of the temperature difference, are generated by the phase transitions of $\mathrm{VO}_{2}$.

The Lissajous $q-\Delta T$ curve of the proposed thermal memristor is shown in Fig. 4. The cycle direction is indicated by the arrows associated to the four behaviors illustrated in Fig. 3. The straight lines in cases 2 and 4 confirm the proportionality $(q=G \Delta T)$ between the heat flux and temperature difference, due to the absence of $\mathrm{VO}_{2}$ phase transitions. The slope $G=M^{-1}$ is the thermal conductance of the system and its value $\left(G(2) \approx 4 \sigma \varepsilon_{m} T_{0}^{3}\right)$ for case 2 is smaller than the one $\left(G(4) \approx 4 \sigma \varepsilon_{i} T_{0}^{3}\right)$ for case 4 , because of $\varepsilon_{m}<\varepsilon_{i}$. The nonlinear behaviors in cases 1 and 3 are due to the $\mathrm{VO}_{2}$ phase transitions driving the emissivity variations displayed in Fig. 3. The absence of these changes near 


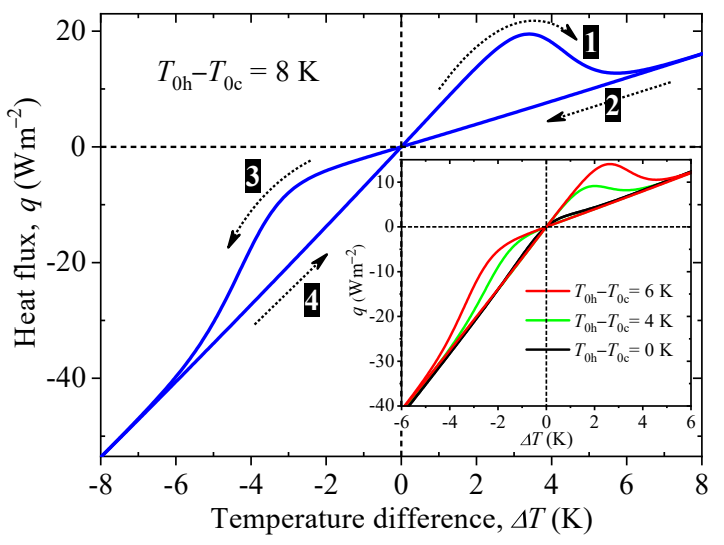

FIG. 4. Lisssajous $q-\Delta T$ curve characterizing the thermal memristor. The regimes $1,2,3$, and 4 correspond to the four characteristic zones highlighed in Fig. 3. Calculations were done for $\theta=8 \mathrm{~K}$ and different hysteresis widths $T_{0 h}-T_{0 c}$.

the extreme values $( \pm 8 \mathrm{~K})$ of $\Delta T$ induces the pinched shape of the Lissajous curve. Note that the amplitude (differences between the curves $1-2$ and $3-4$ ) of this defining curve of the thermal memristor, is driven by the hysteresis $\left(T_{0 h}-T_{0 c}=8 \mathrm{~K}\right)$ of the $\mathrm{VO}_{2}$ emissivity, such that it disappears for $T_{0 h}-T_{0 c}=0 \mathrm{~K}$, as shown in the inset of Fig. 4. This amplitude also increases with the emissivity contrast $\varepsilon_{i} / \varepsilon_{m}$ of $\mathrm{VO}_{2}$, as shown in Fig. S2 of the supplementary material. These facts make evident that the existence of the Lissajous $q-\Delta T$ curve is determined by the combined impact of the thermal hysteresis $\left(T_{0 h} \neq T_{0 c}\right)$ and emissivity contrast $\left(\varepsilon_{i} / \varepsilon_{m} \neq 1\right)$ of the involved PCM. This is confirmed by the area of the loops $1-2$ and $3-4$, which are equal and proportional to $\left(\varepsilon_{i}-\varepsilon_{m}\right) \sinh \left[\beta\left(T_{0 h}-T_{0 c}\right) / 2\right]$, as shown in the supplementary material. This latter relation establishes the material requirements to optimize the performance and applications of the proposed thermal memristor.

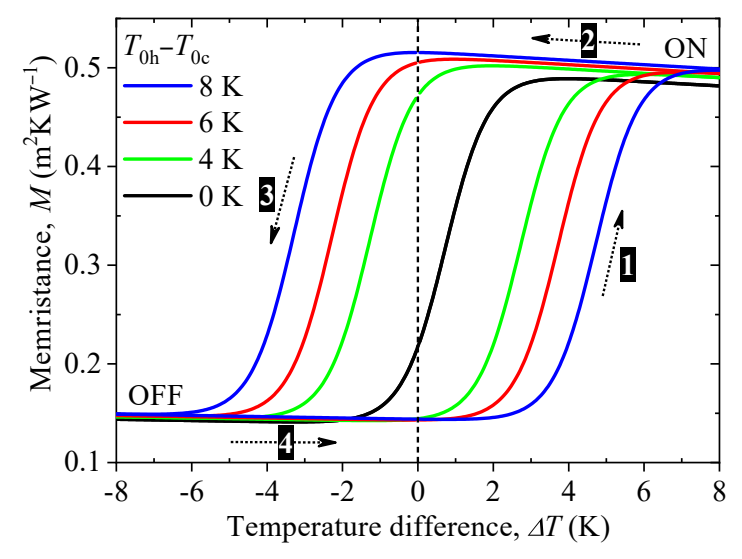

FIG. 5. Closed loops of the thermal memristance associated to the Lissajous curves shown in Fig. 4. The regimes 1, 2,3 , and 4 correspond to the four characteristic time zones highlighed in Fig. 3.
Figure 5 shows the thermal memristances $M$ related to the Lissajous $q-\Delta T$ curves displayed in Fig. 4, as functions of the temperature difference $\Delta T$. The linear portions of the top (2) and bottom (4) lines correspond to the regimes without a phase transition of $\mathrm{VO}_{2}$, while the complementary right (1) and left (3) lines results from its phase changes, in agreement with Figs. 3 and 4. The height of the $M-\Delta T$ loops is determined by the $\mathrm{VO}_{2}$ emissivity contrast $\varepsilon_{i} / \varepsilon_{m}=3.6$, which defines the ON/OFF states of the memristance (Fig. 5) and is higher than the one (1.6) of a conductive memristor [36]. The width of the memristance loop increases with the hysteresis $T_{0 h}-T_{0 c}>0$, such that it dissapears for $T_{0 h}-T_{0 c}=0$, in agreement with Fig. 4. In addition, the closed $M-\Delta T$ loops are similar to those of the electrical memristance [32], which indicates that what is done with electrical currents through the electrical memristor, could also be done with thermal currents, by means of the thermal memristor. For instance, the thermal memristor could be used as a binary memory, in which the $\mathrm{ON}$ and $\mathrm{OFF}$ values of the memristance (Fig. 5) are chosen to code the binary states 0 and 1 , as is done with its electrical counterpart [32]. In addition, given that the proposed radiative memristor can be scaled down to nanometric dimensions through near-field radiation, this device has great potential for developing thermal memories that could complement the electronic flash ones.

Finally, we point out that although the Lissajous $q-\Delta T$ curve reported in this letter has been obtained with one PCM, we anticipate that this memristor effet can also be achieved with more complex systems involving a non-PCM with oscilating temperature (supplementary material) or two PCMs with temperatures properly modulated in time with equal or different frequencies. Furthermore, the development of the radiative memristor in the near field, would allow not only its miniaturization, but also the modulation of its memristance with the separation distance and steady-state temperature component $T_{0}$ of its terminals, due to the strong dependence of the heat flux on these two parameters[51]. Radiative thermal memristors could thus be useful to tailor the heat transfer at different spatial scales.

In summary, the proof of principle of a radiative thermal memristor has been demonstrated, by exploiting the thermal hysteresis of the emissivity of a phase change material exchanging radiative heat with a phase invariable one. For the case of $\mathrm{VO}_{2}$, it has been shown that the Lissajous $q-\Delta T$ curve of the memristor yields a memristance, whose temperature variations in a closed loop are determined by the hysteresis width of $\mathrm{VO}_{2}$ and the ratio of emissivities for its insulating and metallic phases. Considering the unique thermal properties of the proposed thermal memristor, the obtained results can open new research avenues in the development of thermal neurons, energy storage, and thermal management. 
* jose.ordonez@cnrs.pprime.fr

† younes.ezzahri@univ-poitiers.fr

[1] B. Li, J. Lan, and L. Wang, Phys. Rev. Lett. 95, 104302 (2005).

[2] B. Hu, L. Yang, and Y. Zhang, Phys. Rev. Lett. 97, $124302(2006)$.

[3] N. Yang, N. Li, L. Wang, and B. Li, Phys. Rev. B 76, 020301 (2007).

[4] L. Wang and B. Li, Physics World 21, 27.

[5] J. Hu, X. Ruan, and Y. P. Chen, Nano Letters 9, 2730 (2009).

[6] N. Yang, G. Zhang, and B. Li, Appl. Phys. Lett. 95, 033107 (2009).

[7] T. Ruokola, T. Ojanen, and A.-P. Jauho, Phys. Rev. B 79, 144306 (2009).

[8] C. R. Otey, W. T. Lau, and S. Fan, Phys. Rev. Lett. 104, 154301 (2010).

[9] S. Basu and M. Francoeur, Applied Physics Letters 98, 113106 (2011).

[10] H. Iizuka and S. Fan, Journal of Applied Physics 112, 024304 (2012).

[11] L. Zhu, C. R. Otey, and S. Fan, Appl. Phys. Lett. 100, 044104 (2012).

[12] Y. Yang, S. Basu, and L. Wang, Appl. Phys. Lett. 103, 163101 (2013).

[13] B. Li, L. Wang, and G. Casati, Phys. Rev. Lett. 93, 184301 (2004).

[14] M. Terraneo, M. Peyrard, and G. Casati, Phys. Rev. Lett. 88, 094302 (2002).

[15] D. Segal, Phys. Rev. Lett. 100, 105901 (2008).

[16] P. Ben-Abdallah and S.-A. Biehs, Appl. Phys. Lett. 103, 191907 (2013).

[17] E. Nefzaoui, J. Drevillon, Y. Ezzahri, and K. Joulain, Appl. Opt. 53, 3479 (2014).

[18] J. Ordonez-Miranda, J. M. Hill, K. Joulain, Y. Ezzahri, and J. Drevillon, J. Appl. Phys. 123, 085102 (2018).

[19] J. Ordonez-Miranda, Y. Ezzahri, and K. Joulain, Phys. Rev. E 95, 022128 (2017).

[20] B. Li, L. Wang, and G. Casati, Appl. Phys. Lett. 88, 143501 (2006).

[21] H. Prod'homme, J. Ordonez-Miranda, Y. Ezzahri, J. Drevillon, and K. Joulain, J. Appl. Phys. 119, 194502 (2016).

[22] P. Ben-Abdallah and S.-A. Biehs, Phys. Rev. Lett. 112, 044301 (2014).

[23] K. Joulain, J. Drevillon, Y. Ezzahri, and J. OrdonezMiranda, Phys. Rev. Lett. 116, 200601 (2016).

[24] I. Latella, O. Marconot, J. Sylvestre, L. G. Fréchette, and P. Ben-Abdallah, Phys. Rev. Applied 11, 024004 (2019).

[25] K. Joulain, Y. Ezzahri, J. Drevillon, and P. BenAbdallah, Appl. Phys. Lett. 106, 133505 (2015).

[26] J. Ordonez-Miranda, Y. Ezzahri, J. Drevillon, and K. Joulain, J. Appl. Phys. 119, 203105 (2016).

[27] L. Wang and B. Li, Phys. Rev. Lett. 99, 177208 (2007).

[28] V. Kubytskyi, S.-A. Biehs, and P. Ben-Abdallah, Phys.
Rev. Lett. 113, 074301 (2014).

[29] K. Ito, K. Nishikawa, H. Iizuka, and H. Toshiyoshi, Appl. Phys. Lett. 105, 253503 (2014).

[30] A. Fiorino, D. Thompson, L. Zhu, R. Mittapally, S.A. Biehs, O. Bezencenet, N. El-Bondry, S. Bansropun, P. Ben-Abdallah, E. Meyhofer, and P. Reddy, ACS Nano 12, 5774 (2018).

[31] K. Ito, K. Nishikawa, and H. Iizuka, Appl. Phys. Lett. 108, 053507 (2016).

[32] R. Tetzla, Memristors and Memristive Systems (Springer, New York, 2014).

[33] L. O. Chua, IEEE Trans. Circuit Theory 18, 507 (1971).

[34] D. B. Strukov, G. S. Snider, D. R. Stewart, and R. S. Williams, Nature 453, 80 (2008).

[35] P. Ben-Abdallah, AIP Advances 7, 065002 (2017).

[36] F. Yang, M. P. Gordon, and J. J. Urban, J. Appl. Phys. 125, 025109 (2019).

[37] J. Ordonez-Miranda, Y. Ezzahri, K. Joulain, J. Drevillon, and J. J. Alvarado-Gil, Phys. Rev. B 98, 075144 (2018).

[38] A. S. Barker, H. W. Verleur, and H. J. Guggenheim, Phys. Rev. Lett. 17, 1286 (1966).

[39] M. Rini, A. Cavalleri, R. W. Schoenlein, R. López, L. C. Feldman, R. F. Haglund, L. A. Boatner, and T. E. Haynes, Opt. Lett. 30, 558 (2005).

[40] M. M. Qazilbash, M. Brehm, B.-G. Chae, P.-C. Ho, G. O. Andreev, B.-J. Kim, S. J. Yun, A. V. Balatsky, M. B. Maple, F. Keilmann, H.-T. Kim, and D. N. Basov, Science 318, 1750 (2007).

[41] M. M. Qazilbash, M. Brehm, G. O. Andreev, A. Frenzel, P.-C. Ho, B.-G. Chae, B.-J. Kim, S. J. Yun, H.-T. Kim, A. V. Balatsky, O. G. Shpyrko, M. B. Maple, F. Keilmann, and D. N. Basov, Phys. Rev. B 79, 075107 (2009).

[42] K. Joulain, Y. Ezzahri, J. Drevillon, B. Rousseau, and D. D. S. Meneses, Opt. Express 23, A1388 (2015).

[43] J. Yoon, H. Kim, X. Chen, N. Tamura, B. S. Mun, C. Park, and H. Ju, ACS Appl. Mater. Interfaces 8, 2280 (2016).

[44] M. F. Modest, Radiative Heat Transfer (Academic Press, New York, 2013).

[45] S. Lee, K. Hippalgaonkar, F. Yang, J. Hong, C. Ko, J. Suh, K. Liu, K. Wang, J. J. Urban, X. Zhang, C. Dames, S. A. Hartnoll, O. Delaire, and J. Wu, Science 355, 371 (2017).

[46] J. A. Ramirez-Rincon, C. L. Gomez-Heredia, A. Corvisier, J. Ordonez-Miranda, T. Girardeau, F. Paumier, C. Champeaux, F. Dumas-Bouchiat, Y. Ezzahri, K. Joulain, O. Ares, and J. J. Alvarado-Gil, J. Appl. Phys. 124, 195102 (2018).

[47] C. L. Gomez-Heredia, J. A. Ramirez-Rincon, J. OrdonezMiranda, O. Ares, J. J. Alvarado-Gil, C. Champeaux, F. Dumas-Bouchiat, Y. Ezzahri, and K. Joulain, Sci. Rep. 8, 8479 (2018).

[48] J. Ordonez-Miranda, Y. Ezzahri, J. Drevillon, and K. Joulain, Phys. Rev. Applied 6, 054003 (2016).

[49] J. Bodzenta, J. B. Burak, M. Nowak, M. Pyka, M. Szaajko, and M. Tanasiewicz, Dent. Mater. 22, 617 (2006).

[50] F. Cervantes-Alvarez, J. D. Macias, and J. J. AlvaradoGil, J. Phys. D: Appl. Phys. 51, 065302 (2018).

[51] I. Latella, R. Messina, J. M. Rubi, and P. Ben-Abdallah, Phys. Rev. Lett. 121, 023903 (2018). 